\title{
The Sounds of Levitating Water Droplets
}

\section{Leidenfrost drops suspended above a hot surface by a thin layer of vapor emit periodic sounds in a similar way to pipe organs.}

By Erika K. Carlson

$\square$ prinkle water onto a very hot pan, and you may notice that the droplets evaporate surprisingly slowly. They stick around because of what's called the Leidenfrost effect-a thin layer of vapor forms between the droplets and the hot surface, insulating them from the heat, and keeping them from boiling off immediately. What you're less likely to notice is that droplets of water in this Leidenfrost regime emit periodic sounds, or beats. Now, Tanu Singla and Marco Rivera of Morelos State University and the Monterrey Institute of Technology and Higher Education in Mexico have analyzed these sounds and deduced their origin [1].

Singla and Rivera stumbled on the phenomenon while investigating other aspects of the Leidenfrost effect. To explore the nature of the noises, they recorded audio and video footage of water droplets placed onto a hot, concave aluminum surface. While emitting sounds, the droplets oscillated as pulsing stars whose points moved radially in and out. By measuring the frequency of a droplet's oscillations, the researchers calculated the frequency of the vibrations in the layer of vapor underneath,
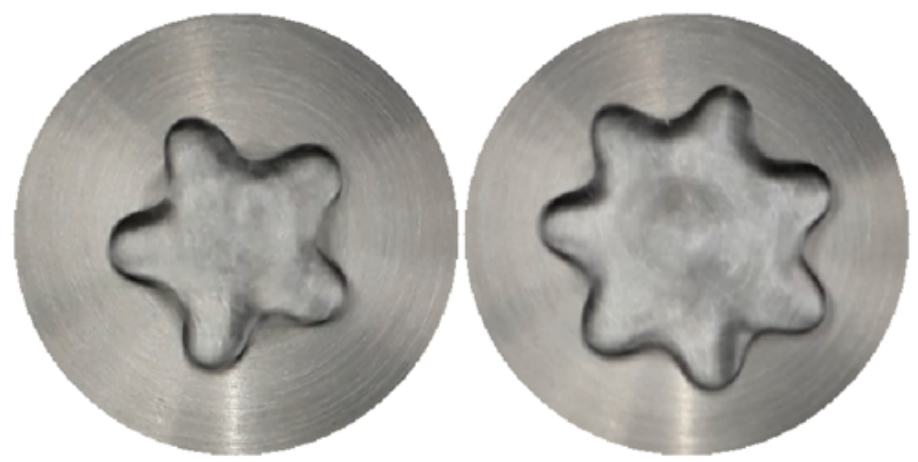

Credit: T. Singla and M. Rivera [1] using a relation found by previous studies. They determined that this vapor-layer frequency matched the period of the beats, and they therefore concluded that vapor escaping from beneath the droplet was responsible for producing the periodic sounds.

The researchers also found that the frequency of the sounds made by a droplet depended on the droplet's size-following the model of an organ pipe, whose tone depends on the velocity of sound and the length of the pipe. This implies that the sound production mechanism in a Leidenfrost droplet is similar to that of a wind instrument.

Erika K. Carlson is a Corresponding Editor for Physics based in New York City.

\section{REFERENCES}

1. T. Singla and M. Rivera, "Sounds of Leidenfrost drops," Phys. Rev. Fluids 5, 113604 (2020). 